\section{Successful Women Ceramic and Glass Scientists and Engineers: 100 Inspirational Profiles}

Lynnette D. Madsen

Wiley, 2016

640 pages, \$65.00 (e-book \$52.99) ISBN 978-1-118-73360-8
$\mathrm{O}$ fall the successful female scientists, most outside of their field would only recognize a handful of their names. Madame Curie is world renowned, and others, such as Rosalind Franklin, are now getting their due credit. However, the history of most sciences is nearly devoid of famous women.

Madsen makes an attempt to change the trajectory of women's place in the physical sciences in this book. Her stated purpose is to remedy the underrepresentation and underappreciation of women in physical sciences by profiling the careers of a diverse range of 100 contemporary women in a "narrow area of materials science and engineering to make a point [that] there are plenty of successful women [scientists] out there!" Other objectives of the book are to help the careers of the women featured and also to attract and retain more women into the physical sciences. From her perch as a program director in materials research at the National Science Foundation in the United States, Madsen is well positioned to see such a broad range of research and how women are making an impact.
The book is organized into 100 chapters, listing the women in alphabetical order. Each profile is structured in the same manner and contains each woman's name, position, birthplace, "tags" for their interest (administration, industry, etc.), and personal information, such as nationality, race, and number of children. The front page also contains a photo of each woman at a young age. Also included are the publication/invention record for each (plus her h-index) and her proudest career moment to date. This is followed by her academic credentials, research expertise, list of awards, a short biography of her early life and career history, and three most cited publications. Finally, her perspective on challenges in science and being a woman in science are described. Each profile wraps up with some final words of wisdom and a recent photo. All is neatly summarized in four to six pages for each profile.

Plodding through the stories of women's lives in alphabetical order in the same format, I soon felt like I was looking through resumes for a new hire (a "binder of women"). I became distracted by h-factors and citation counts, and after skimming the awards, I wondered why some bothered to list teaching awards and symposia that they had organized. The advice to women varied from blaming men for keeping them down to simply being grateful for the opportunity to work in science. The words of wisdom were filled with standard platitudes: work hard, build relationships, and do not be afraid. Absent was the sense of inspiration that I had been hoping for and have found in other books on women in science.

While Madsen's project was a tremendous effort, I think it could have been improved upon with more aggressive editing and perhaps highlighting half as many women, with a focus on different categories (e.g., university, industry, inventors, policy, etc.) rather than an alphabetical listing. Shorter award lists and more personal stories might have made the chapters more engaging.

However, in the aggregate, Madsen may have achieved her goal, as the book is highly web searchable. This book is a useful tool for those trying to evaluate these 100 women for more awards or for those looking for conference speakers. While not the most poetic book, nor the stuff of bedtime reading, Madsen has created a tome such that no one can deny that women are now major contributors to the physical sciences.

Reviewer: Karen Swider Lyons researches fuel-cell and battery materials and their integration into naval systems in Alexandria, Va., USA.

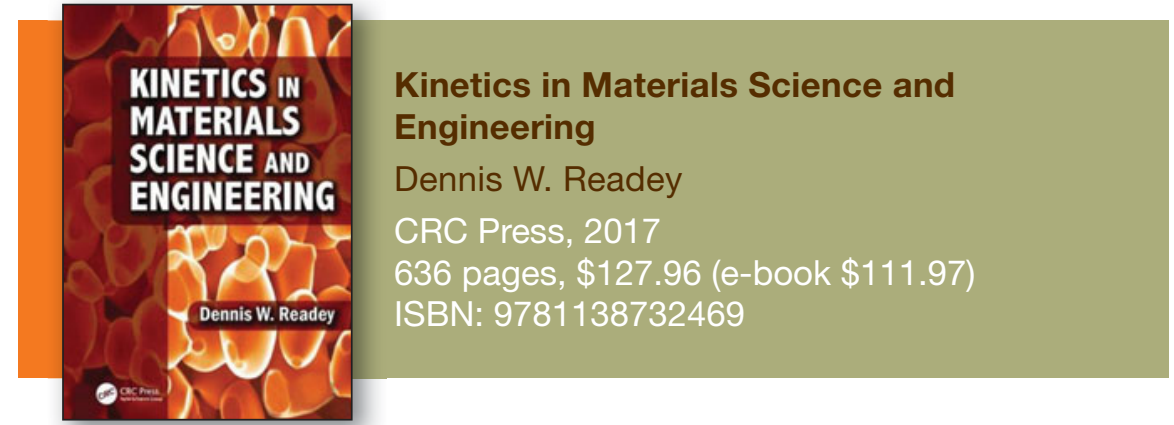

This book is intended for materials science and engineering undergraduate students who have taken a course on kinetics in physical chemistry. It intends to give in-depth knowledge in the area of kinetics of the formation and reactivity of a variety of materials. The book came out as a result of rich experiences gained by the author in teaching a course on materials science and engineering for more than 30 years in three institutions. The preface of the book summarizes the main features of the book, with an emphasis on treatment at atomistic and macroscopic levels, solutions to diffusion-controlled processes, derivation of the Boltzmann distribution equation, treating the glass transition as a relaxation process, and the Kirkendall effect applied to metals, polymers, and soft materials. Some of these topics include industrial examples. 
The book is divided into five sections. The first section introduces the reader to kinetics in materials science and engineering with an overview of its relationship to thermodynamics. The second section on reaction kinetics is divided into four chapters, with coverage of processes in materials, second order and multiple reactions, and temperature dependence of the reaction rate as applied to homogeneous and heterogeneous reactions. The next section covers phase transformations by discussing surface energies from considerations involving the existence of broken bonds in metals and polymers. This section contains extensive discussion on freezing point depression of small particles, interfacial energies, surface segregation, and surface tension.

The fourth section includes five chapters: (1) fundamentals of diffusion, (2) atomistics of diffusion, (3) steady-state diffusion, (4) solutions to Fick's second law, and (5) diffusion with finite boundaries. This section discusses the thermodynamics of phase transitions, reconstructive transformations, and nucleation and growth applied to homogeneous and heterogeneous cases, as well as diffusion phenomenon. The last section considers different types of fluxes, forces, and interdiffusion, with a treatment on nonideal thermodynamic behavior of solid and liquid solutions. The utility of the book is enhanced by a subject index and two appendices containing symbols.

The book provides every derivation and brings out the kinetic processes in materials science and engineering in an understandable way. As a teacher who has taught and is teaching courses in materials science and engineering and who has performed research in the area of electrochemical kinetics, I find this book extraordinary in all respects in giving in-depth mathematical derivations. However, the emphasis given on electrochemical kinetics applied to transient techniques using Fick's laws is minimal, except for dealing with ion conductance.

This is an excellent book, and although it should not be used as the primary text for a kinetics course (no mention of the fundamentals and applications of kinetics, such as pulse radiolysis; fluorescence; photolysis; temperature-jump, pressure jump, or stopped flow techniques; electron spin resonance; or nuclear magnetic resonance), I strongly recommend it as supplemental text for a general course introducing materials science and engineering at the graduate level.

Reviewer: K.S.V. Santhanam is a professor in the School of Chemistry and Materials Science at Rochester Institute of Technology, USA.

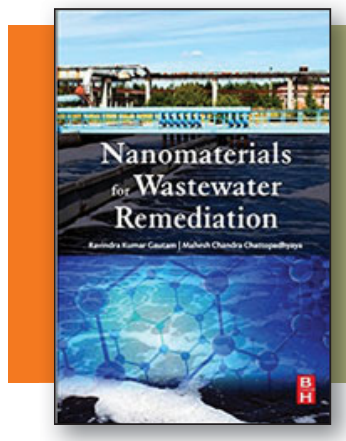

Nanomaterials for Wastewater Remediation

Ravindra Kumar Gautam and Mahesh Chandra Chattopadhyaya

Butterworth-Heinemann, 2016

366 pages, \$170.00 (e-book \$170.00)

ISBN 9780128046098
$\mathrm{T}$ his book is a comprehensive overview of the advances in water remediation processes via nanotechnology. It covers most of the nanomaterials that are presently being used, as well as nanomaterials that are still under investigation, such as magnetic nanoparticles, bimetallic nanoparticles, and carbon- and alumina-based structures. References are ample, as the book is written for all types of readers, not only those in the scientific community. For novices in water remediation, this book provides basic information on the field and is a good textbook for students; for experts, the book provides updates on state-of-the-art methods.

Chapter 1 introduces various nanomaterials that are promising agents for water remediation. It also emphasizes the lack of cost-effectiveness and risk assessment for the current nanomaterials and their by-products. Chapter 2 focuses on industrial pollutants and contemporary water remediation solutions, such as reverse osmosis, advanced oxidation techniques (further discussed in chapter 3 ), nanosorbtion (with an emphasis on graphene-based nanosorbents, further detailed in chapters 4-6), and magnetic nanomaterials. Chapter 3 addresses catalytic nanomaterials capable of generating highly reactive $\mathrm{OH}^{-}$radicals via photochemical, sonochemical, electrochemical, and photo-Fenton oxidation processes.

Chapters 4-6 are dedicated to the growing field of graphene-based nanosorbents that are efficient in capturing heavy metals, such as $\mathrm{Hg}$ and $\mathrm{Pb}$, along with organic dyes. Graphene or reduced graphene-based organic or inorganic composites and their efficiency on toxins and various organic and inorganic pollutants are reviewed and systematized. Chapter 5 reviews kinetic models of graphene-based sorbents. Also, Langmuir, Temkin, and Freundlich isotherms are discussed for various nanosorbents, and functionalized graphene is mentioned as being efficient in adsorbing particular heavy metals. Chapter 6 discusses magnetic nanoparticles combined with graphene-based nanocomposites and photocatalytic composites for sorption of dyes.

Chapter 7 makes the case for magnetic nanoparticles as efficient nanosorbents on their own, rather than being coupled with graphene. This chapter also discusses the stability of these nanomaterials in terms of the $\mathrm{pH}$ of the solution, as well as their synthesis and morphology. Magnetite and maghemite nanoparticles and their composites in the removal of arsenic are given particular attention. Chapter 8 describes the potential of layered double hydroxides possessing high anion exchange capacity in the removal of organic and inorganic 